\title{
HOLOMORPHIC LEFSCHETZ FIXED POINT FORMULA
}

\author{
BY V. K. PATODI ${ }^{1}$ \\ Communicated by Michael Atiyah, December 27, 1972
}

1. Let $X$ be an $n$-dimensional complex analytic manifold and $\varphi: X \rightarrow X$ a holomorphic map. Let $\Omega$ be the sheaf of germs of holomorphic functions on $X$ and $H^{i}(X, \Omega)$ the $i$ th cohomology group of $X$ with coefficients in the sheaf $\Omega$. The map $\varphi$ defines endomorphisms, $H^{i}(\varphi)$ of $H^{i}(X, \Omega), i \geqq 0$. Let $L(\varphi)$ be the Lefschetz number defined by

$$
L(\varphi)=\sum_{i=0}^{n}(-1)^{i} \operatorname{trace} H^{i}(\varphi)
$$

We are concerned with the problem of computing $L(\varphi)$.

REMARK. Let $\mathrm{G}$ be a compact Lie group acting on $X$ as a group of holomorphic diffeomorphisms and $\varphi \in G$. The problem in this case has been solved by Atiyah and Singer, see [2]. Also in the case $\varphi$ has isolated fixed points, the problem was solved in the nondegenerate case (see $\$ 2$ for definition) by Atiyah and Bott in [1] and by Toledo and Tong im [6] and [7] in the degenerate case.

2. The statement of main theorem. Let $X_{\varphi}$ be the fixed point set of the map $\varphi, X_{\varphi}=\{x \in X$ s.t. $\varphi(x)=x\}$. We start by stating the conditions under which we have been able to compute the Lefschetz number $L(\varphi)$.

$\left(C_{1}\right) X_{\varphi}$ is a complex analytic submanifold of $X$ and moreover with this complex analytic structure, $X_{\varphi}$ is a Kähler manifold.

Let us write $X_{\varphi}$ as a finite union of closed connected submanifolds of $X$ :

$$
X_{\varphi}=\bigcup_{i=1}^{N} Y_{i}
$$

Let $\lambda_{1}^{i}, \ldots, \lambda_{m_{i}}^{i}$ be the eigenvalues of the endomorphism $\left(\varphi_{*}\right)_{z}$ of $T_{z}(X)$, $z \in Y_{i}$, with multiplicities $n_{1}^{i}, \ldots, n_{m_{i}}^{i}$; eigenvalues $\lambda_{j}^{i}$ are independent of $z \in Y_{i}$ because of the holomorphic nature of the situation. If 1 is an eigenvalue of the map $\varphi_{*}$ we take $\lambda_{1}^{i}=1$.

The vector bundles $\left.T(X)\right|_{Y_{i}}$ decompose as a direct sum of holomorphic vector subbundles $E_{j}^{i}\left(1 \leqq j \leqq m_{i}\right)$ whose fibres $\left(E_{j}^{i}\right)_{z}$ are defined by:

$$
\left(E_{j}^{i}\right)_{z}=\left\{v \in T_{z}(X) \text { s.t. }\left(\varphi_{*}-\lambda_{j}^{i} I\right)^{n_{j}^{i}} v=0\right\} .
$$

We now state our other conditions.

$\left(\mathrm{C}_{2}\right)$ The fixed points are nondegenerate: 1 is an eigenvalue of

AMS (MOS) subject classifications (1970). Primary 58G10, 53C65; Secondary 32A99.

${ }^{1}$ Supported in part by National Science Foundation grant GP-36418X. 
$\varphi_{*}: T_{z}(X) \rightarrow T_{z}(X)$ iff the dimension $r_{i}$ of $Y_{i}$ is greater than zero and in case $r_{i}>0, n_{1}^{i}=r_{i}$.

$\left(\mathrm{C}_{3}\right)$ There exists a hermitian metric $h$ in $T(X)$ such that

(a) $h\left(v_{z}, w_{z}\right)=0$ if $v_{z} \in T_{z}\left(Y_{i}\right), w_{z} \in \sum_{\lambda_{j} \neq 1}\left(E_{j}^{i}\right)_{z}, z \in Y_{i}$.

(b) If $\Omega$ is the canonical 2-form associated to $h$, then, $(d \Omega)_{z}=(\nabla d \Omega)_{z}=0$, $z \in X_{\varphi}, \nabla$ is the hermitian connection defined by $h$.

$\left(\mathrm{C}_{4}\right)$ The vector bundles $E_{j}^{i}$ decompose as

$$
E_{j}^{i}=\sum_{k=1}^{N_{i j}} E_{j k}^{i}
$$

such that each $E_{j k}^{i}$ is a holomorphic subbundle and $E_{j N_{i j}}^{i}=0$ and $\varphi_{*}-\lambda_{j}^{i} I$ maps $E_{j k}^{i}$ into $E_{j k+1}^{i}, k \geqq 1$.

It is not very natural to impose conditions $\left(C_{3}\right)$ and $\left(C_{4}\right)$. We however have simple conditions which always guarantee the conditions $\left(C_{3}\right)$ and $\left(C_{4}\right)$ :

(1) Let $X$ be a Kähler manifold and $\varphi$ preserves the metric. Then the conditions $\left(C_{1}\right)$ to $\left(C_{4}\right)$ are all satisfied.

(2) The condition $\left(C_{3}\right)$ is satisfied if for positive integers $i(1 \leqq i \leqq N)$ such that $r_{i}>1\left(r_{i}=\right.$ dimension of $\left.Y_{i}\right)$ the eigenvalues $\lambda_{j}^{i}$ satisfy the following inequality: $\lambda_{j}^{i} \lambda_{j^{\prime}}^{i} \neq 1$ for $j, j^{\prime} \geqq 2$.

(3) Suppose that $X$ is a Kähler manifold and $H^{0,1}\left(Y_{i},\left(\Sigma_{j} E_{j}^{i}\right)^{*}\right)=0$ for $1 \leqq i<N$ such that $r_{i}>1$, where given a vector bundle $\xi$, $\xi^{*}$ denotes the dual bundle. Then the condition $\left(\mathrm{C}_{3}\right)$ holds.

(4) If the maps $\left(\varphi_{*}-\lambda_{j}^{i} I\right)^{k}: E_{j}^{i} \rightarrow E_{j}^{i}, 1 \leqq k \leqq n_{j}^{i}, 1 \leqq i \leqq N$ such that $r_{i}>1$, are of constant rank, then the condition $\left(C_{4}\right)$ holds.

We note that if each $r_{i}$ is either $n-1$ or is at most one, then the condition $\left(C_{4}\right)$ is satisfied and $\left(C_{3}\right)$ is also satisfied if $\varphi_{*}: T_{z}(X) \rightarrow T_{z}(X)$ does not have eigenvalue -1 for $z \in Y_{i}$ such that $r_{i}=n-1$.

We now proceed to state our theorem. Let $C_{1}, C_{2}, \ldots, C_{n_{j}^{i}}$ be Chern classes of $E_{j}^{i}$ and consider the formal factorization:

The formal power series

$$
1+\sum t^{k} C_{k}=\prod_{k=1}^{n_{j}^{i}}\left(1+t x_{k}\right)
$$

$$
\mathscr{U}_{j}^{i}=\prod_{k}\left(\frac{1-\lambda_{j}^{i} \exp \left(-x_{k}\right)}{1-\lambda_{j}^{i}}\right)^{-1}, \quad \lambda_{j}^{i} \neq 1,
$$

is symmetric in $x_{i}$ 's and hence can be expressed as a polynomial in $C_{k}$ 's.

THEOREM 1. If the conditions $\left(\mathrm{C}_{1}\right)$ to $\left(\mathrm{C}_{4}\right)$ are satisfied, then

$$
L(\varphi)=\sum_{i=1}^{N}\left(\prod_{\lambda_{j}^{i} \neq 1}\left(1-\lambda_{j}^{i}\right)^{n_{j}^{i}}\right)^{-1} \times\left\{\left(\prod_{\lambda_{j} \neq 1} \mathscr{U}_{j}^{i}\right) \mathscr{T}\left(Y_{i}\right)\right\}\left[Y_{i}\right],
$$


where the class $\mathscr{U}_{j}^{i}$ is defined by (3), $\mathscr{T}\left(Y_{i}\right)$ is the Todd class of $T\left(Y_{i}\right)$ and given a class $\alpha \in H^{*}\left(Y_{i}, C\right),\{\alpha\}\left[Y_{i}\right]$ denotes the evaluation of the $2 r_{i}$ th component of $\alpha\left(r_{i}=\right.$ complex dimension of $\left.Y_{i}\right)$ on the fundamental cycle of $Y_{i}$ determined by its natural orientation.

3. Outline of the proof. We first observe that under the conditions $\left(\mathrm{C}_{1}\right)$ to $\left(\mathrm{C}_{4}\right)$ there exists a hermitian metric $h$ in $T(X)$, the tangent bundle of $X$, such that the condition $\left(C_{3}\right)$ is satisfied and furthermore $h\left(v_{z}, w_{z}\right)=0$ if $z \in Y_{i}, v_{z} \in\left(E_{j k}^{i}\right)_{z}, w_{z} \in\left(E_{j^{\prime} k^{\prime}}^{i}\right)_{z}$, the pair $(j, k) \neq\left(j^{\prime}, k^{\prime}\right)$, where the bundles $E_{j k}^{i}$ are the ones occurring in the decomposition (2) of condition $\left(\mathrm{C}_{4}\right)$.

Let $\Lambda^{0, q}$ be the bundle of differential forms of type $(0, q)$ with the metric induced from $h, d_{\bar{z}}$ be the canonical operator (exterior differentiation with respect to $\bar{z}$ ) from $C^{\infty}\left(\Lambda^{0, q}\right)$ to $C^{\infty}\left(\Lambda^{0, q+1}\right), 0 \leqq q \leqq n$, and $d_{\bar{z}}^{*}$ be its adjoint. Let $\Delta_{\bar{z}}^{q}=-\left(d_{\bar{z}} d_{\bar{z}}^{*}+d_{\bar{z}}^{*} d_{\bar{z}}\right): C^{\infty}\left(\Lambda^{0, q}\right) \rightarrow C^{\infty}\left(\Lambda^{0, q}\right)$ be the Laplace operator and $e^{q}\left(t, z^{\prime}, z\right)$ be the fundamental solution of the heat operator $\partial / \partial t-\Delta_{\frac{q}{z}}^{q}$.

Now there exists an $\varepsilon>0$ such that the disc bundle $N_{\varepsilon}$ over the fixed point manifold $X_{\varphi}$ defined by

$$
N_{\varepsilon}=\left\{v \in T_{x}(X) \text { s.t. } x \in X_{\varphi} \text { and }\|v\|<\varepsilon\right\},
$$

(where \|\| is defined by the metric) is diffeomorphic to a neighborhood of $X_{\varphi}$ in $X$. The form $\left(\varphi_{z^{\prime}}^{*} e^{q}\left(t, z^{\prime}, z\right)\right)_{z^{\prime}=z^{*}} 1$ defines under this diffeomorphism a form on $N_{\varepsilon}$, which we shall denote by $E^{q}(t, z)$.

There is a natural map $\pi_{*}: C^{\infty}\left(\Lambda T^{*}\left(N_{\varepsilon}\right)\right) \rightarrow C^{\infty}\left(\Lambda T^{*}\left(X_{\varphi}\right)\right)$ such that $\int \psi_{1} \wedge \pi_{*}\left(\psi_{2}\right)=\int \pi^{*}\left(\psi_{1}\right) \wedge \psi_{2}, \quad \psi_{2} \in C^{\infty}\left(\Lambda T^{*}\left(N_{\varepsilon}\right)\right), \quad \psi_{1} \in C^{\infty}\left(\Lambda T^{*}\left(X_{\varphi}\right)\right)$, $\pi: N_{\varepsilon} \rightarrow X$ being the projection.

Let $\psi_{t}^{q}=\pi_{*}\left(E^{q}(t, z)\right)$. We have the following proposition:

Proposition 2. $H(\varphi)=\sum_{q=0}^{n}(-1)^{q} \int_{X_{\varphi}} \psi_{t}^{q}$, as $t \downarrow 0$, the forms $\psi_{t}^{q}$ turn out to be independent of $\varepsilon>0$ (as $t \downarrow 0)$.

Moreover we have the following theorem:

THEOREM 3. (Local form of Lefschetz fixed point formula.) We have at each point $z \in Y_{i}, 1 \leqq i \leqq N$,

$$
\begin{aligned}
\sum_{q=0}^{n}(-1)^{q} \psi_{t}^{q}(z)= & \left(\prod_{\lambda_{j} \neq 1}\left(1-\lambda_{j}^{i}\right)^{n_{j}}\right)^{-1} \\
& \times 2 r_{i} \text { th component of }\left[\left(\prod_{\lambda_{j} \neq 1} \mathscr{U}_{j}^{i}\right) \mathscr{T}\left(Y^{i}\right)\right](z)+O(t),
\end{aligned}
$$

as $t \downarrow 0$,

where $\mathscr{U}_{j}^{i}$ 's are the characteristic classes defined in $\$ 2$ and here represented as a differential form by Andre Weil's homomorphism, the connections used in $T\left(Y_{i}\right), E_{j}^{i}$ are the hermitian connections defined by the hermitian metric. 
Theorem 1 is an immediate consequence of Proposition (2) and Theorem (3). Theorem (3) is of course stronger than Theorem (1). Our proof of Theorem (3) depends on the method developed in [4] and [5].

REMARK. The results have natural extension to the situation when one considers the Lefschetz number associated to the data: a holomorphic vector bundle $\xi$ over $X$, a holomorphic map $\varphi$ of $X$ into itself and a vector bundle analytic homomorphism $\tilde{\varphi}$ of $\varphi^{*}(\xi)$ into $\xi$.

The author wants to express his thanks to Professors M. F. Atiyah, R. Bott, I. M. Singer and Dr. R. S. Kulkarni for useful discussions.

\section{REFERENCES}

1. M. F. Atiyah and R. Bott, A Lefschetz fixed point formula for elliptic complexes. I and II, Ann. of Math. (2) 86 (1967), 374-407, 88 (1968), 451-491. MR 35 \# 3701; 38 \# 731.

2. M. F. Atiyah and I. M. Singer, The index of elliptic operators. I and III, Ann. of Math. (2) 87 (1968), 484-530; 87 (1968), 546-604. MR 38 \# 5243; 38 \#5245.

3. T. Kotake, The fixed point theorem of Atiyah-Bott via parabolic operators, Comm. Pure Appl. Math. 22 (1969), 789-806.

4. V. K. Patodi, Curvature and the eigenforms of the Laplace operator, J. Differential Geometry 5 (1971), 233-249.

5. - An analytic proof of Riemann-Roch-Hirzebruch theorem for Kähler manifolds, J. Differential Geometry 5 (1971), 251-283.

6. D. Toledo, On the Atiyah-Bott formula for isolated fixed points, J. Differential Geometry (to appear).

7. Yue Lin L. Tong, deRham's integrals and Lefschetz fixed point formula for $d^{\prime \prime}$ cohomology, Bull. Amer. Math. Soc. 78 (1972), 420-422.

School of Mathematics, Institute for Advanced Study, Princeton, New Jersey 08540

School of Mathematics, Tata Institute of Fundamental Research, Bombay-5, India (Current address) 